\title{
Adaptability and Resilience of Academic Radiation Oncology Personnel and Procedures during Covid-
}

\section{Pandemic}

Indra J. Das, ${ }^{1, *}$ John A. Kalapurakal, Jonathan B. Strauss, Brian R. Zawislak, Mahesh Gopalakrishnan, Amishi Bajaj, Bharat B. Mittal

Department of Radiation Oncology, Northwestern Memorial Hospital, Northwestern University Feinberg School of Medicine, 251 East Huron Street, Galter Pavilion, LC-178, Chicago, IL 60611; Indra.das@nm.org *Correspondence: Indra.das@nm.org e-mail@e-mail.com; Tel.: (+1 312-926-4884, IJD)

\begin{abstract}
:
Background: A comprehensive response to the unprecedented SARS-CoV-2 (COVID-19) challenges for public health and its impact on radiation oncology patients and personnel for resilience and adaptability is presented. Methods: The general recommendations included working remotely when feasible, implementation of screening/safety and personal protective equipment (PPE) guidelines, social distancing, regular cleaning of treatment environment, and testing for high-risk patients/procedures. All teaching conferences, tumor boards, and weekly chart rounds were conducted using a virtual platform. Additionally, specific recommendations were given to each section to ensure proper patient treatments. The impact of these measures, especially adaptability and resilience, were evaluated through specific questionnaire surveys. Results: These comprehensive COVID-19 related measures resulted in most staff expressing a consistent level of satisfaction in regards to personal safety, maintaining a safe work environment, continuing quality patient care and continuing educational activities during the pandemic. There was a significant reduction in patient treatments and on-site patient visits with an appeciable increase in the number of telemedicine e-visits. Conclusions: Survey results demonstrated substantial adaptability and resilience, including in the rapid recovery of departmental activities during the reactivation phase. In the event of a future public health emergency, the measures implemented may be adopted with good outcomes by radiation oncology departments across the globe.
\end{abstract}

Keywords: Covid-19; Radiation Oncology; Operation; Survey; Adaptability; Resilience

\section{Introduction}

About a century ago, the influenza pandemic of 1918 ("the Spanish Flu") infected nearly half a billion and killed nearly 50 million people worldwide [1]. A similar outbreak was recognized in December 262019 by SARS-CoV-2 (COVID-19) and has since rapidly spread to become a pandemic posing critical challenges for public health, global economy and clinical medicine [2, 3]. As of March 2021, COVID-19 has infected over 126 million people worldwide and resulted in 2.8 million deaths (COVID-19 Statistics, 2021). Unfortunately, hospitals worldwide were not immediately prepared to cope with the impact of this pandemic, scrambling to prioritize resources for identifying, testing, and managing critically ill COVID-19 patients [4]. The disease spreads rapidly to airways with inflammation of respiratory system within a few days with appearance of pneumonia and sepsis. The chest imaging using $x$-rays and CT scans shows patchy or diffuse asymmetric 
airspace opacities, a distinct ground glass patchy pattern in lungs [5-7]. A few medical options to treat patients had a minimal gain [8]. Most elective procedures in the hospitals were suspended to support the pandemic health crisis.

Without a talisman and lack of effective treatments, infected patients were managed with supportive measures, and stay-at-home orders and closure of all non-essential businesses were required for society-at-large. Social distancing and use of personal protective equipment (PPE) were recommended [4, 9]. Essential healthcare services like urgent care centers and hospitals remained open, while non-emergent surgical and diagnostic procedures were canceled. Cancer treatments were postponed when it was deemed not safe when weighed against risk of contracting COVID-19 infection [10, 11]. Radiation oncology communities attempted to treat cancer patients with variable precautions, with altered practice as necessitated by local needs [11, 12]. There has been suggestion of lowdose radiation to the whole lungs for COVID-19 treatment [13] but consequences and opposition of radiation is also debated [14].

The multi-institutional survey conducted by the German group illustrated 46 the European experience with radiotherapy treatment in the face of the COVID-19 pandemic [15]. This study describes the comprehensive response undertaken by radiation oncology staff in the face of COVID-19 at an academic institution in the United States (USA), seeks to understand the impact on personnel and procedures, and demonstrates the adaptability and resilience noted following implementation of certain mitigating measures.

\section{Materials and Methods}

\subsection{Mitigating Procedures}

The comprehensive response detailed below was developed jointly by the hospital COVID-19 task force and radiation oncology leadership. These teams held regular teleconferences initially daily and then weekly to manage the changing crisis with proper recommendations.

\subsection{General Recommendations}

- Hospital infection control policies were strictly followed

- Everyone entering the department was monitored for temperature and screened for symptoms such as fever, cough, or chest pain. If febrile or symptomatic, employees were directed to receive COVID-19 testing

- Staff treating COVID-19 positive patients were required to wear full PPE, gloves, mask, gowns, goggles or face-shields)

- Everyone was required to wear masks and gloves, and wash their hands with soap/hand sanitizers before and after patient contact

- Visitors were allowed in the department only when needed

- All treatment devices and surfaces were cleaned with disinfectant wipes after each treatment

- Exam room doors remained open for contactless operation and air exchange

- Consultations and follow-ups were held using telemedicine 
- Covid-19 positive patients were treated at the end of the day on a designated machine

- A two week quarantine period was mandated when a member living in the staff/patient's home tested positive

- Patients were tested negative for special procedures (brachytherapy, Gamma knife and SBRT)

- Children requiring sedation for irradiation were tested weekly

- Laptop computers and database access were provided to staff for remote work

- All teaching conferences, tumor boards, weekly chart rounds were virtually conducted using teleconferencing

\subsection{Physicians}

- $\quad$ Patients were triaged into three groups; (1) Patients with radiation emergencies, (2) Patients whose treatment could not be postponed and (3) Patients with cancers where treatment could be safely postponed. In general, treatment was only given for groups 1 and 2

- Shorter treatment courses were used when feasible [10-12]

- Only one on-call physician was in the department daily, others were in the clinic only once a week for patient's on-treatment visits

\subsection{Nursing}

- Two nurses were required to be in the clinic

- Nurses were responsible for maintaining a clean and contamination-free clinic and observing

- $\quad$ social distancing among patients

\subsection{Physicists}

- At least two physicists were on site for 2-3 days each week

- Additional staffing were required on site for special procedures (Two physicists each for Brachytherapy and Gamma Knife and one for the SBRT)

- Treatment plans, chart checks were performed by off-site physicists

- Calibration, machine maintenance and QA were performed after hours

\subsection{Dosimetrists}

- Two dosimetrists were available on site daily

- Every dosimetrist was provided laptop computers to facilitate remote work

- Communications were maintained as needed via email, phone and teleconferencing

\subsection{Radiation Therapists}

- A two-week rotation with 3-days/week for treatment was adopted

- When patient volumes decreased to 50\%, they were placed in hospital's "labor pool" to allow

- $\quad$ them for the full-time salary 
- Instructed to remain in their assigned rooms to minimize cross exposure

- After each treatment, they are required to disinfect every treatment device and surfaces

\subsection{Residents and Students}

- Students were instructed to stay at home unless needed

- One on-call resident was required to be in the clinic each week, while the others were allowed to work from home

- All clinical and educational activities were performed remotely

\subsection{Laboratory Research}

- Laboratory research was halted during the Covid-19 period except data analyses, computational work, maintenance of cell lines and freezer stocks

- Research staff were instructed to stay at home

- Computational research support was available to staff and faculty

\subsection{Impact of Departmental Response}

- For this report, the pandemic timelines were: pre-COVID-19 (February, 29, 2020), COVID-19 (3/1/2020 until 5/10/2020) and the Reactivation phase (5/11/2020 until $5 / 29 / 2020$ ). The impact of comprehensive measures on personnel and procedures was determined by the following:

- Questionnaire survey response from all staff based on Institutional Review Board (IRB) exempt status

- Census of departmental activities (consultations, treatments and attendance during teaching and clinical conferences)

- Covid-19 infection rates among staff and patients

- Rates of normalization of activities during the Reactivation phase

\section{Results}

\subsection{Questionnaire Response}

The responses to a section-specific questionnaires sent to staff are summarized in Figure 1 . The compliance to survey were $100 \%(n=73)$ as every clinical member of the department participated in the survey privately and remotely. The scores ranged from $0-10$, with 0 indicating the least and 10 the highest satisfaction. While questionnaires were section-specific, three questions were common to the entire department. These were analyzed with results shown in Figure 2 indicating consistent level of satisfaction with the proposed measures aimed at ensuring personal safety, maintaining a safe work environment, continuing quality patient care and providing educational activities during the pandemic. The data from this survey and institutional survey conducted before pandemic are nearly identical indicating that satisfaction with the management and patient care were not impacted due to pandemic indicating a high degree of resilience in the staff. 

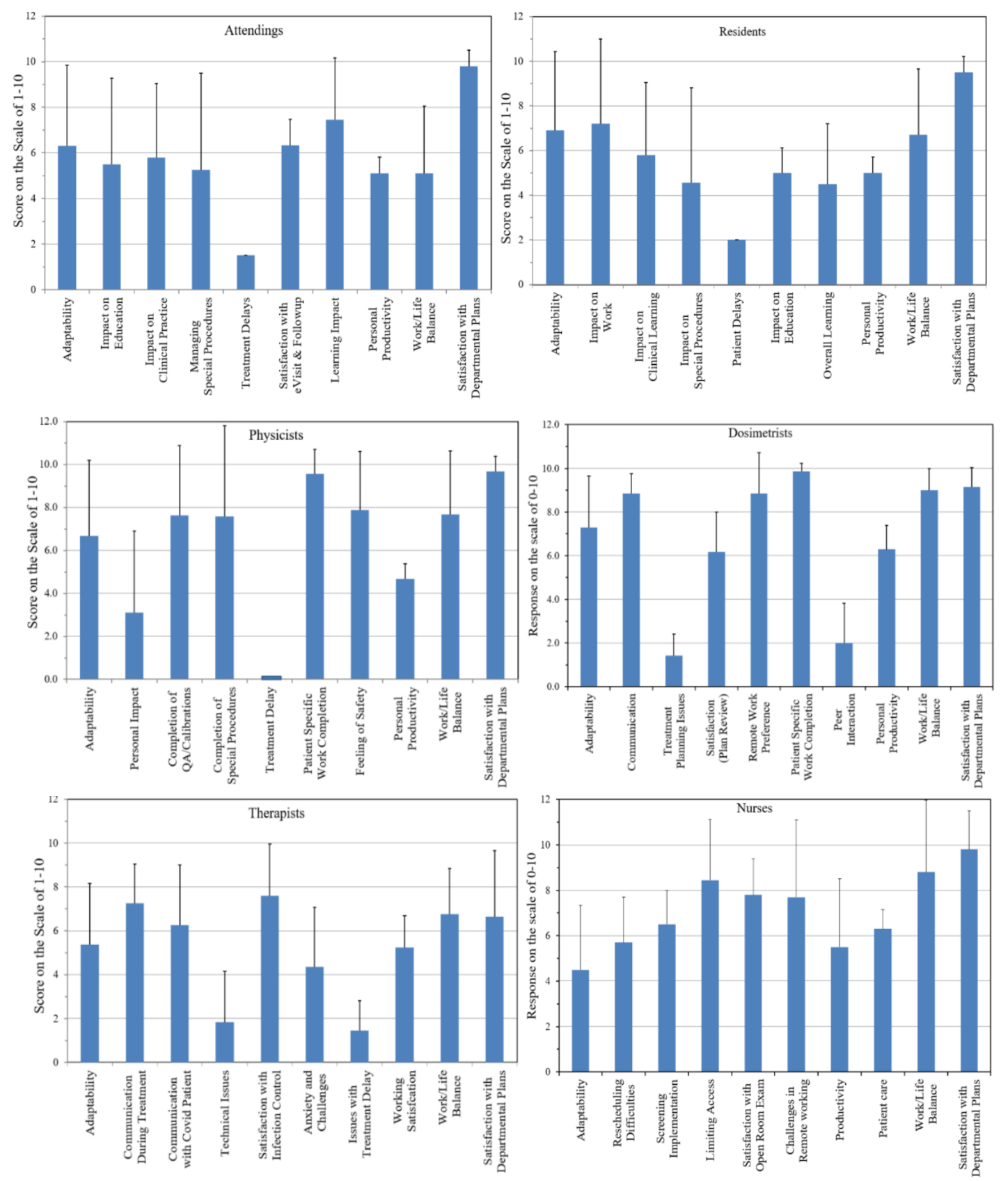

Figure 1. Questionnaire responses from each section of the department. Questions in each group are not identical. 


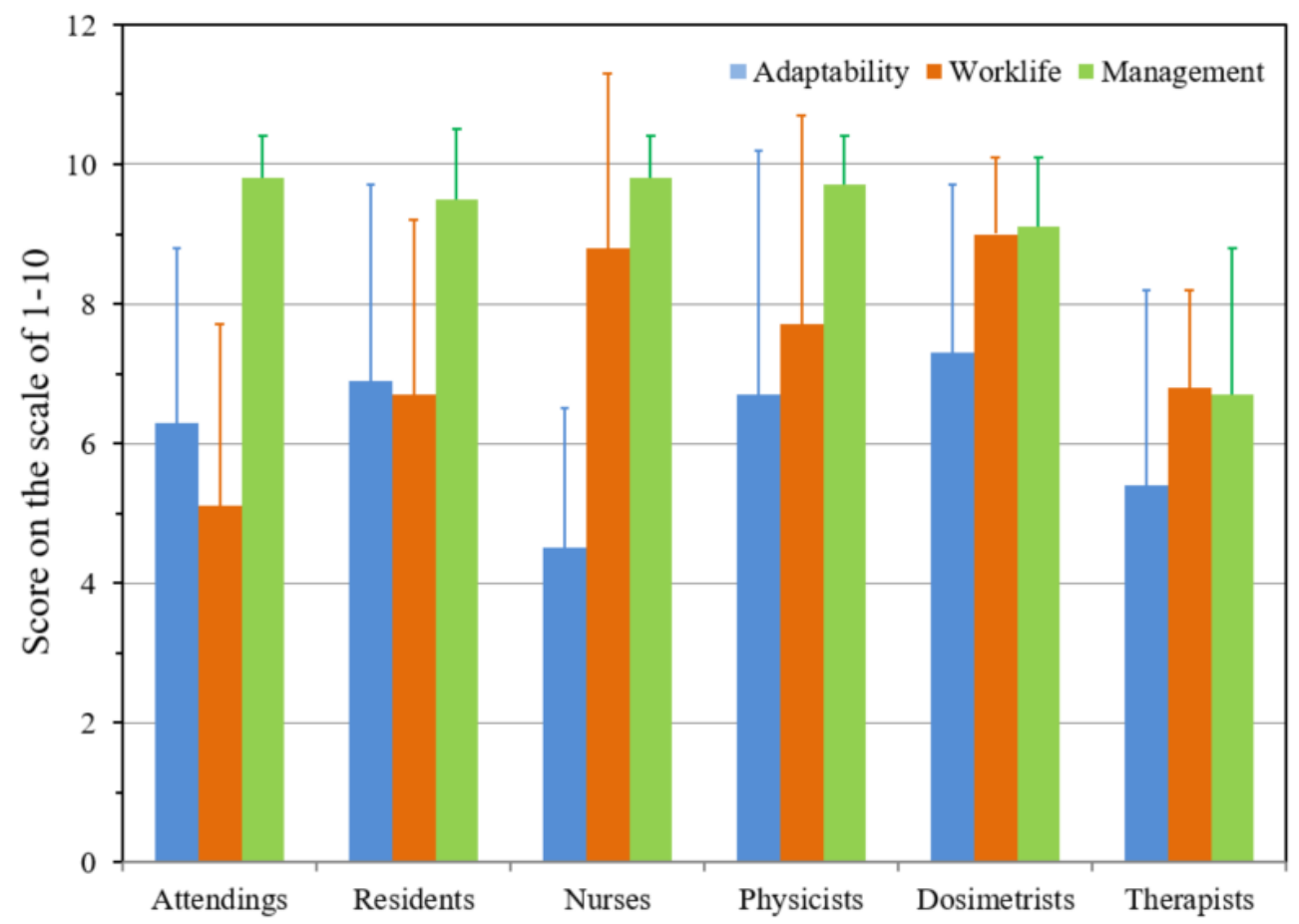

Figure 2: Analysis of the three common questions for all staff members. Please note the adaptability is variable in each group depending on the contacts with patients.

\subsection{Census of departmental activities}

The census of patient consultations, simulation and treatments (Fig. 3) shows the negative impact of the pandemic on clinical activities. The number of patients treated dropped significantly $( \pm 20 \%)$ during the Covid-19 period and the on-site consults and follow-ups fell rapidly to nearly $1 / 3$ during Covid- 19 phase. However, this was compensated by a significant increase in the number of telemedicine e-visits. The use of telemedicine has enabled consistent levels of participation by staff and residents at pre-Covid levels for all educational conferences (journal clubs, didactic lectures) and weekly chart rounds.
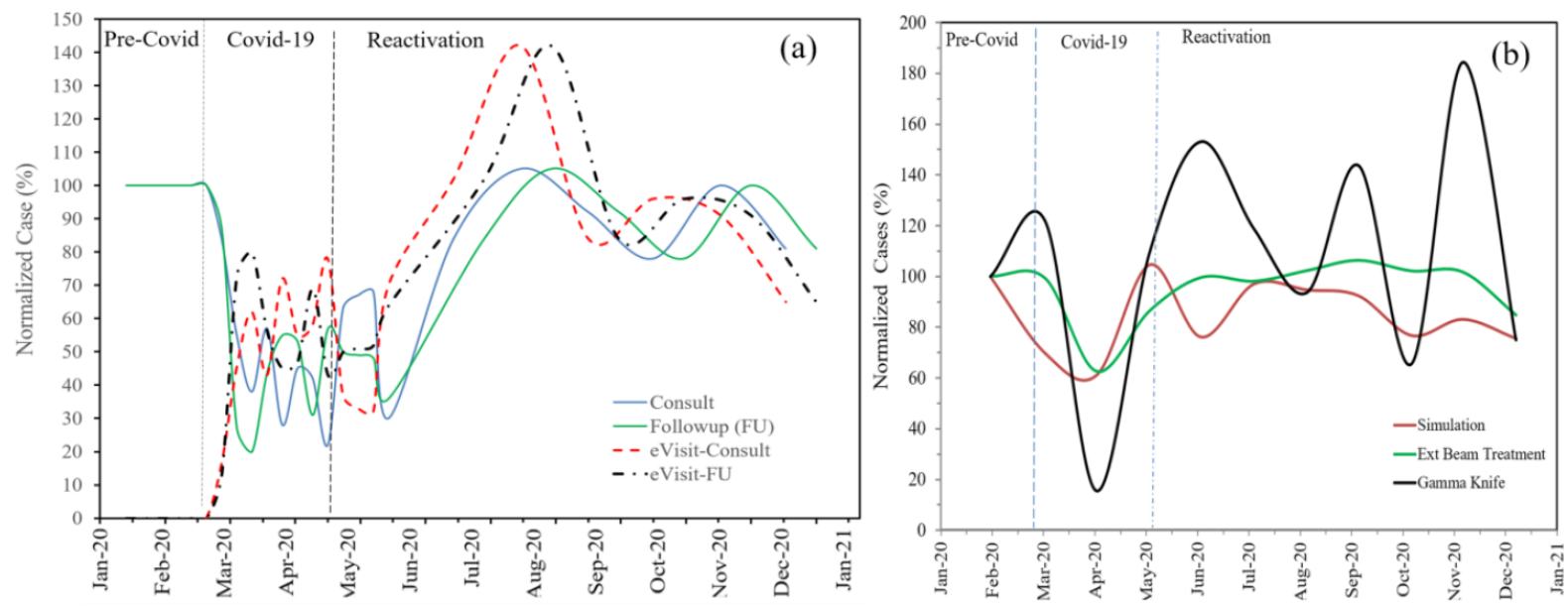
Figure 3. (a) Pattern of consults, follow-ups and respective e-Visits, (b) Census of patient simulations, treatments, and Gamma Knife. Note that during Covid-19 most activities were significantly reduced to nearly $20 \%$ of the normal load.

\subsection{Covid-19 Infection rates}

During this reporting period, none of the staff or students have developed symptoms or signs of Covid- 19 infection. Six patients with tested positive Covid-19 were treated according to standard protocols. None of these patients died. Two patients required radiation treatment while they were Covid-19 positive. During reactivation phase a Covid-19 surge in the USA took place where we had $8 \%$ positive patients treated without any staff being infected.

\subsection{Census of Activities during the Reactivation Phase}

The high morale and resilience of our departmental clinical staff and the support of the hospital authorities resulted in the rapid recovery of departmental activities during the reactivation phase (Fig. 3 ).

\section{Discussions}

In the face of a global pandemic with a virus lacking effective treatment, it is natural for clinical staff who are concerned for their own and family's well-being, to be also fearful for having to choose between their clinical obligations and the stay-at-home orders issued by state governments. The comprehensive measures described in this report were implemented with regular feedback and collaboration among all staff, to effectively deal with the pandemic and mitigate its impact on personnel and procedures. The questionnaire responses indicated that there was general satisfaction with overall goal of ensuring personal safety and providing a good work environment very similar to the data provided by Reuter-Oppermann et al [15]. There was a significant reduction in the number of patients treated during the Covid-19 phase as patients were triaged depending on the urgency of their diagnosis as described earlier [10-12]. The use of telemedicine was immensely helpful in enabling our patients to continue to receive medical advice and also it was critical in mitigating the potential negative impact on resident education and other departmental conferences [16, 17]. The closure of all laboratories and cessation of basic research has adversely affected animal research. The low rates of symptomatic infections among our patients and staff lends supports the value of social distancing, use of PPE and maintaining a contamination-free clinical environment. A significant weakness of this report is the lack of periodic and comprehensive testing for Covid-19 infection for staff and patients. This was due to the national shortage of testing kits and the strategic decision to direct such resources to symptomatic staff and patients [18].

This study is limited to an academic department with multiple section. However, one can extrapolate this data for a community practices. Another caveat is the location of our center, which is in down town of a very large metropolis, and our experience may not directly reflect for the rural centers. 


\section{Conclusions}

This report describes the lessons learned from a comprehensive strategy for all staff and procedures in an academic radiation oncology department during the Covid-19 pandemic. These recommendations were successful as shown by the survey in maintaining staff morale and safety, delivering treatment safely to patients and maintaining the quality of resident education during the pandemic. With proper precautions radiation oncology patients are successfully treated as shown by the survey indicating adequate resilience and adaptability in the face of Covid-19. These measures can be easily adopted by any radiation therapy department with good outcomes in the event of a second wave or future pandemics.

Author Contributions: This study is not funded by any organization.

Funding: This research received no external funding.

Institutional Review Board Statement: IRB exempt approved status used in this study.

Informed Consent Statement: There is no patient involved.

Acknowledgments: We greatly acknowledge administrative and technical support from Daniel Sheedy, Amy Stevenson, Paul Fischer, Heather Mallet, Gayle Woloschak, Tatjana Paunesku.

Conflicts of Interest: The authors declare no conflict of interest.

\section{References}

1. Belser JA, Tumpey TM. The 1918 flu, 100 years later. Science. 2018;359(6373):255. Epub 2018/01/20. doi: 10.1126/science.aas9565. PubMed PMID: 29348212.

2. Fauci AS, Lane HC, Redfield RR. Covid-19 - Navigating the Uncharted. N Engl J Med. 2020;382(13):1268-9. Epub 2020/02/29. doi: 10.1056/NEJMe2002387. PubMed PMID: 32109011; PubMed Central PMCID: PMCPMC7121221.

3. Li Q, Guan X, Wu P, Wang X, Zhou L, Tong Y, et al. Early Transmission Dynamics in Wuhan, China, of Novel Coronavirus-Infected Pneumonia. N Engl J Med. 2020;382(13):1199-207. Epub 2020/01/30. doi: 10.1056/NEJMoa2001316. PubMed PMID: 31995857; PubMed Central PMCID: PMCPMC7121484.

4. Timmis K, Brüssow H. The COVID-19 pandemic: some lessons learned about crisis preparedness and management, and the need for international benchmarking to reduce deficits. Environ Microbiol. 2020;22(6):1986-96. Epub 2020/04/23. doi: 10.1111/1462-2920.15029. PubMed PMID: 32319151; PubMed Central PMCID: PMCPMC7264722.

5. Kooraki S, Hosseiny M, Myers L, Gholamrezanezhad A. Coronavirus (COVID-19) Outbreak: What the Department of Radiology Should Know. J Am Coll Radiol. 2020;17(4):447-51. Epub 2020/02/25. doi: 10.1016/j.jacr.2020.02.008. PubMed PMID: 32092296; PubMed Central PMCID: PMCPMC7102595.

6. Hope MD, Raptis CA, Shah A, Hammer MM, Henry TS. A role for CT in COVID-19? What data really tell us so far. Lancet. 2020;395(10231):1189-90. Epub 2020/04/01. doi: 10.1016/s0140-6736(20)30728-5. PubMed PMID: 32224299.

7. Hosseiny M, Kooraki S, Gholamrezanezhad A, Reddy S, Myers L. Radiology Perspective of Coronavirus Disease 2019 (COVID-19): Lessons From Severe Acute Respiratory Syndrome and Middle East Respiratory Syndrome. AJR Am J Roentgenol. 2020;214(5):1078-82. Epub 2020/02/29. doi: 10.2214/ajr.20.22969. PubMed PMID: 32108495. 
8. Beigel JH, Tomashek KM, Dodd LE, Mehta AK, Zingman BS, Kalil AC, et al. Remdesivir for the Treatment of Covid-19 - Final Report. N Engl J Med. 2020;383(19):1813-26. Epub 2020/05/24. doi: 10.1056/NEJMoa2007764. PubMed PMID: 32445440; PubMed Central PMCID: PMCPMC7262788.

9. Hsiang S, Allen D, Annan-Phan S, Bell K, Bolliger I, Chong T, et al. The effect of large-scale anti- contagion policies on the COVID-19 pandemic. Nature. 2020;Jun 8. doi: 10.1038/s41586-020-2404-8. Online ahead of print. Epub 2020/06/09. doi: 10.1038/s41586-020-2404-8. PubMed PMID: 32512578.

10. Kuderer NM, Choueiri TK, Shah DP, Shyr Y, Rubinstein SM, Rivera DR, et al. Clinical impact of COVID-19 on patients with cancer (CCC19): a cohort study. Lancet. 2020;395(10241):1907-18. Epub 2020/06/01. doi: 10.1016/s0140-6736(20)31187-9. PubMed PMID: 32473681; PubMed Central PMCID: PMCPMC7255743.

11. Combs SE, Belka C, Niyazi M, Corradini S, Pigorsch S, Wilkens J, et al. First statement on preparation for the COVID-19 pandemic in large German Speaking University-based radiation oncology departments. Radiat Oncol. 2020;15(1):74. Epub 2020/04/09. doi: 10.1186/s13014-020-01527-1. PubMed PMID: 32264908; PubMed Central PMCID: PMCPMC7136995.

12. Simcock R, Thomas TV, Estes C, Filippi AR, Katz MA, Pereira IJ, et al. COVID-19: Global radiation oncology's targeted response for pandemic preparedness. Clin Transl Radiat Oncol. 2020;22:55-68. Epub 2020/04/11. doi: 10.1016/j.ctro.2020.03.009. PubMed PMID: 32274425; PubMed Central PMCID: PMCPMC7102593.

13. Ameri A, Ameri P, Rahnama N, Mokhtari M, Sedaghat M, Hadavand F, et al. Low-Dose Whole-Lung Irradiation for COVID-19 Pneumonia: Final Results of a Pilot Study. Int J Radiat Oncol Biol Phys. 2021;109(4):859-66. Epub 2020/12/06. doi: 10.1016/j.ijrobp.2020.11.065. PubMed PMID: 33278503; PubMed Central PMCID: PMCPMC7709599.

14. Das IJ, Kalapurakal JA, Mittal BB. Caution warranted for low-dose radaition therapy for Covid-19. Br J Radiol. 2021;93:20200466. doi: Doi:10.1259/BJR.20200466.

15. Reuter-Oppermann M, Müller-Polyzou R, Wirtz H, Georgiadis A. Influence of the pandemic dissemination of COVID-19 on radiotherapy practice: A flash survey in Germany, Austria and Switzerland. PloS one. 2020;15(5):e0233330. Epub 2020/05/22. doi: 10.1371/journal.pone.0233330. PubMed PMID: 32437381; PubMed Central PMCID: PMCPMC7241763.

16. Hollander JE, Carr BG. Virtually Perfect? Telemedicine for Covid-19. N Engl J Med. 2020;382(18):1679-81. Epub 2020/03/12. doi: 10.1056/NEJMp2003539. PubMed PMID: 32160451.

17. Hollander JE, Davis TM, Doarn C, Goldwater JC, Klasko S, Lowery C, et al. Recommendations from the First National Academic Consortium of Telehealth. Popul Health Manag. 2018;21(4):271-7. Epub 2017/10/05. doi: 10.1089/pop.2017.0080. PubMed PMID: 28976250.

18. Schneider EC. Failing the Test - The Tragic Data Gap Undermining the U.S. Pandemic Response. N Engl J Med. 2020;May 15. doi: 10.1056/NEJMp2014836. Online ahead of print. Epub 2020/05/16. doi: 10.1056/NEJMp2014836. PubMed PMID: 32412704 . 